\title{
Protocolo de intervenção do Departamento de Ortopedia e Traumatologia de um hospital universitário de alta complexidade para enfrentamento da pandemia de COVID-19*
}

\section{Intervention Protocol of the Orthopedics and Traumatology Department of a High-Complexity University Hospital to Cope with the COVID-19 Pandemic}

\author{
Ewerton Borges de Souza Lima1® ${ }^{10}$ Paulo Santoro Belangero ${ }^{1}$ Guilherme Guadagnini Falótico ${ }^{10}$ \\ Nacime Salomão Barbachan Mansur ${ }^{10}$ Marcus Vinicius Malheiros Luzo ${ }^{10}$ Fernando Baldy dos Reis ${ }^{10}$ \\ ${ }^{1}$ Departamento de Ortopedia e Traumatologia, Escola Paulista de
Medicina, Universidade Federal de São Paulo (Unifesp), São Paulo,
SP, Brasil \\ Rev Bras Ortop 2020;55(3):269-277.

\begin{abstract}
Endereço para correspondência Ewerton Borges de Souza Lima, MD, Escola Paulista de Medicina, Universidade Federal de São Paulo (Unifesp), Rua Três de Maio 130, apto. 183, Vila Clementino, São Paulo - SP, 04044-020, Brasil (e-mail: ewertonbslima@gmail.com).
\end{abstract}

\section{Resumo \\ Palavras-chave \\ - COVID-19 \\ - gestão hospitalar \\ - ortopedia \\ - pandemias \\ - segurança ocupacional \\ - síndrome respiratória aguda grave}

Objetivo Descrever os protocolos implementados para adequar a equipe ortopédica à pandemia de COVID-19 e, secundariamente, avaliar o comportamento de variáveis hospitalares e departamentais frente à implementação das medidas.

Métodos Estudo retrospectivo com descrição de três protocolos: 1) Redução do risco de contágio dos pacientes e profissionais de saúde; 2) Adaptação das atividades laborais, acadêmicas e científicas; e 3) Adaptação do tratamento ortopédico frente à pandemia. Foram analisadas variáveis hospitalares e departamentais como: número de pacientes atendidos no pronto-socorro e ambulatórios, pacientes internados, casos suspeitos e confirmados nos pacientes e na equipe ortopédica, mudança das atividades de ensino do modo presencial para videoconferência, e número cirurgias. Foi comparado o período $\mathrm{A}$, de 03 a 16 de março, com o período $\mathrm{B}$, de 17 a 30 de março, que corresponde à implementação dos protocolos.

Resultados Houve diminuição do número de pacientes atendidos e do número de pacientes internados. Foram notificados dois suspeitos e um confirmado. Dos integrantes do departamento, 12 foram suspeitos e 6 confirmados. Manteve-se a frequência semanal das aulas e as reuniões clinico-científicas diminuíram de dezpara três. Houve redução no volume de cirurgias.

\footnotetext{
Trabalho desenvolvido no Departamento de Ortopedia e Traumatologia, Escola Paulista de Medicina, Universidade Federal de São Paulo (Unifesp), São Paulo, SP, Brasil.
}

recebido

14 de Abril de 2020

aceito

20 de Abril de 2020
DOI https://doi.org/

10.1055/s-0040-1712972. ISSN 0102-3616.
Copyright $\odot 2020$ by Sociedade Brasileira License terms de Ortopedia e Traumatologia. Published by Thieme Revinter Publicações Ltda, Rio de Janeiro, Brazil 


\begin{abstract}
Keywords

- COVID-19

- hospital administration

- orthopedics

- pandemics

- occupational health

- severe acute respiratory syndrome

Objective To describe the protocols implemented to adapt the orthopedic team to the COVID-19 pandemic; a secondary objective is to assess the behavior of hospital and departmental variables when implementing such measures.

Methods The present is a retrospective study describing three protocols: 1 ) reduction in the risk of infection for patients and healthcare workers; 2) adaptation of work, academic and scientific activities; 3 ) adaptation of the orthopedic treatment during the pandemic. We analyzed hospital and departmental variables, including the number of patients seen in the emergency room and outpatient clinic, the number of hospitalized patients, suspected and confirmed cases in patients and orthopedic team members, changes in teaching activities from on-site attendance to video conferencing and number of surgeries. Period A, from March 3 to 16, was compared with period B, from March 17 to 30, 2020, which corresponded to the implementation of the protocols. Results There was a decrease in the number of outpatients and inpatients. One confirmed and two suspected cases were notified. Among the departmental members, there were 12 suspected and 6 confirmed cases. The weekly frequency of classes was maintained, while the clinical-scientific meetings decreased from ten to three. In addition, the number of surgeries was reduced.

Conclusions The present study demonstrated the feasibility and efficiency of the rapid implementation of intervention protocols in the midst of the COVID-19 pandemic. The protocols focused on reducing the risk of infection for patients and healthcare professionals, adapting work, academic and scientific activities, and modifying the orthopedic treatment. With the application of the protocols, the healthcare, academic and scientific activities remained functional, prioritizing measures to confront the COVID-19 pandemic.
\end{abstract}

Conclusões $\mathrm{O}$ presente estudo demonstrou a viabilidade e a eficiência da rápida implementação de protocolos de intervenção em meio ao cenário de pandemia por COVID-19. Os protocolos focaram na redução do risco de contágio dos pacientes e profissionais de saúde, na adaptação das atividades laborais, acadêmicas e científicas, e na adaptação do tratamento ortopédico. Com sua aplicação, mantiveram-se as atividades assistencial, acadêmica e científica funcionantes, priorizando-se medidas de enfrentamento à COVID-19.

\section{Introdução}

Em 31 de dezembro de 2019, a China relatou um grupo de casos de pneumonia com causa não identificável que posteriormente seria diagnosticada como síndrome respiratória aguda grave coronavírus 2 (severe acute respiratory syndrome coronavirus 2 , SARS-CoV-2, em inglês). ${ }^{1}$ A nova enfermidade foi denominada doença do coronavírus 19 (COVID-19). ${ }^{2} \mathrm{Em}$ decorrência do aparecimento de casos em outros países do mundo, a Organização Mundial da Saúde (OMS), declarou no dia 11 de março de 2020 uma pandemia de SARS-CoV-2. ${ }^{3}$ Globalmente, até 30 março de 2020 foram confirmados 784.738 casos e mais de 37.774 óbitos. $^{4}$

No Brasil, o primeiro caso foi confirmado no dia 26 de fevereiro, em São Paulo, de uma pessoa que havia recentemente viajado à Itália. ${ }^{5}$ No dia 17 de março de 2020 foi declarado estado de emergência pelo prefeito de São Paulo, ${ }^{6}$ e no dia 21 do mesmo mês foi declarado estado de calamidade pública pelo governador do estado. ${ }^{7}$
Neste contexto, o Departamento de Ortopedia e Traumatologia (DOT) da Universidade Federal de São Paulo (Unifesp) percebeu a necessidade de criar um Comitê de Gestão de Crise COVID-19 (CGC COVID-19) para rapidamente criar protocolos de intervenção que adequassem as ações de toda a equipe ortopédica de seu hospital referência à pandemia.

O objetivo primário deste estudo é descrever os protocolos implementados para adequar a equipe ortopédica à pandemia de COVID-19 e, secundariamente, avaliar o comportamento de variáveis hospitalares e departamentais frente à implementação das medidas.

\section{Materiais e Métodos}

O presente estudo foi registrado no Comitê de Ética em Pesquisa/Unifesp sob o número 4560090420. Trata-se de um estudo retrospectivo com descrição dos protocolos criados pelo DOT a partir 17 de março de 2020 e análise de variáveis por estes afetadas. Os dados foram coletados de 
registros administrativos e de prontuários do hospital, e são referentes aos períodos de 3 a 16 de março de 2020 (período A) e de 17 a 30 de março de 2020 (período B), ou seja, 14 dias antes e depois do início da intervenção.

O CGC/COVID-19 foi criado em 17 de março de 2020 e é composto por 7 integrantes do DOT. Foi responsável pelo desenvolvimento de protocolos de intervenção à pandemia, tendo como base as publicações recentes sobre o tema. ${ }^{8-17}$ Os protocolos instituídos foram os seguintes:

- Protocolo 1: redução do risco de contágio dos pacientes e profissionais de saúde;

- Protocolo 2: adaptação das atividades laborais, acadêmicas e científicas;

- Protocolo 3: adaptação do tratamento ortopédico frente à pandemia.

As intervenções adotadas por cada protocolo estão descritas nas - Tabelas 1, 2 e 3. As orientações de Equipamentos de Proteção Individual (EPIs) para cada setor hospitalar estão listadas na - Tabela 4.

Os protocolos e suas atualizações foram divulgados diariamente pelo CGC COVID-19 por meio do aplicativo WhatsApp (Facebook, Inc., Menlo Park, CA, EUA) a todo o DOT, composto por 57 residentes, 41 especializandos e aproximadamente 150 professores, técnicos administrativos em edu- cação (TAEs) e médicos colaboradores, totalizando aproximadamente 250 membros.

As intervenções do protocolo 1 foram avaliadas pelas seguintes variáveis:

- Variação do número de pacientes atendidos no prontosocorro (PS) e nos ambulatórios;

- Variação do volume de pacientes internados na disciplina de Ortopedia e Traumatologia avaliada por meio da porcentagem de ocupação dos leitos da enfermaria;

- Número de pacientes transferidos para a enfermaria da disciplina de Infectologia;

- Variação do número de pessoas nas visitas acadêmicas;

- Número de dias sem EPI adequado no PS; e

- Casos suspeitos, casos confirmados, óbitos e profissionais de saúde contaminados (confirmados e suspeitos).

As intervenções do protocolo 2 foram avaliadas pelas seguintes variáveis:

- Número de ortopedistas treinados para atendimento de síndromes gripais;

- Reorganização das escalas de plantão dos chefes e plantonistas;

- Variação do número semanal de aulas para residência e reuniões científicas;

Tabela 1 Protocolo 1-redução do risco de contágio dos pacientes e profissionais de saúde

\begin{tabular}{|c|c|}
\hline $\begin{array}{l}\text { Pronto- } \\
\text { Socorro }\end{array}$ & $\begin{array}{l}\text { - Instalação de recipientes com álcool em gel nos locais de atendimento; } \\
\text { - Orientações aos pacientes sobre a pandemia (medidas de higiene, importância do isolamento social, sinais e } \\
\text { sintomas de alerta, quando procurar atendimento médico-hospitalar); } \\
\text { - Redução do número de atendimentos. }\end{array}$ \\
\hline Ambulatório & $\begin{array}{l}\text { - As mesmas medidas do Pronto-Socorro; } \\
\text { - Agendamento apenas de consultas de pós-operatórios recentes ou casos que necessitem de acompanha- } \\
\text { - Remto próximo; } \\
\text { secretários do grupo responsável pelo ambulatório; } \\
\text { - Marcação de consultas com horário espaçado para evitar aglomeração; } \\
\text { - Orientação de apenas um acompanhante por paciente. }\end{array}$ \\
\hline Enfermaria & $\begin{array}{l}\text { - Instalação de recipientes com álcool em gel na entrada dos quartos e em locais estratégicos da enfermaria; } \\
\text { - Orientações aos pacientes sobre a pandemia (medidas de higiene, importância do isolamento social, sinais e } \\
\text { - sintomas de alerta, quando procurar atendimento médico-hospitalar); } \\
\text { - Tentificação e isolamento dos casos suspeitos e confirmados; } \\
\text { - Transfe de swab nasal e tomografia de tórax para casos suspeitos; } \\
\text { - Redução do número de pessoas nas visitas acadêmicas; } \\
\text { - Redução no número de pacientes por quarto; } \\
\text { - Colocação de máscara cirúrgica em todos os pacientes. }\end{array}$ \\
\hline $\begin{array}{l}\text { Centro } \\
\text { cirúrgico }\end{array}$ & $\begin{array}{l}\text { - Colocação de máscara cirúrgica em todos os pacientes; } \\
\text { - Atençào à sequência de desparamentação: luvas, seguidas por óculos, avental, e, por fim, máscara; } \\
\text { - Adequação da estrutura do centro cirúrgico e dos procedimentos anestésicos; } \\
\text { - Preparação de uma sala cirúrgica de grande porte para receber pacientes com suspeita ou confirmados para } \\
\text { - Inoença; } \\
\text { - Instituição de pressão negativa na sala cirúrgica e limpeza terminal ao fim de cada cirurgia; } \\
\text { - pacientes; } \\
\text { - Preferência para anestesias com bloqueios periféricos e espinais, de forma a evitar procedimentos que } \\
\text { liberam aerossóis; } \\
\text { - Realização da intubação para anestesia geral, quando necessária, com auxílio de visualizador e apenas um } \\
\text { auxiliar junto ao anestesista no momento do procedimento; treinamento da equipe anestésica para uso de } \\
\text { cubos acrílicos de proteção durante intubação e extubação, visando diminuir a dispersão de aerossóis }\end{array}$ \\
\hline
\end{tabular}


Tabela 2 Protocolo 2-adaptação das atividades laborais, acadêmicas e científicas

\begin{tabular}{|c|c|}
\hline $\begin{array}{l}\text { Ativid } \\
\text { labor }\end{array}$ & $\begin{array}{l}\text { - Reorganização das escalas de plantão dos chefes e plantonistas para se adequar à diminuição da demanda por } \\
\text { atendimentos; } \\
\text { - Orientação aos servidores com mais de } 60 \text { anos e/ou integrantes do grupo de risco para não frequentar o } \\
\text { hospital; } \\
\text { - Liberação dos funcionários do setor administrativo para realizar home-office utilizando comunicação por } \\
\text { telefone, e-mail e WhatsApp; } \\
\text { - Treinamento dos ortopedistas para o atendimento e manejo de síndromes gripais de baixa complexidade, bem } \\
\text { como o manejo e orientação dos pacientes com quadro clínico leve e suspeita de COVID-19 pela equipe da } \\
\text { disciplina de Infectologia e do Serviço de Controle de Infecção Hospitalar. }\end{array}$ \\
\hline & $\begin{array}{l}\text { - Redução do número de pessoas nas visitas acadêmicas; } \\
\text { - Suspensão das aulas e reuniões científicas presenciais da graduação e residência médica por tempo } \\
\text { indeterminado; } \\
\text { - Realização de reuniões científicas e aulas por videoconferência utilizando os aplicativos Google Hangout Meets } \\
\text { ou Zoom após orientação de todos os membros do Departamento de Ortopedia e Traumatologia sobre o seu } \\
\text { uso por meio de cartilha produzida pelo Comitê de Gestão de Crise COVID-19 divulgada pelo aplicativo } \\
\text { WhatsApp; } \\
\text { - Divulgação do cronograma de estudos pelo aplicativo WhatsApp e envio de e-mails para orientação do estudo } \\
\text { domiciliar; }\end{array}$ \\
\hline
\end{tabular}

Tabela 3 Protocolo 3-adaptação do tratamento ortopédico

\begin{tabular}{|c|c|}
\hline $\begin{array}{l}\text { Pronto- } \\
\text { Socorro }\end{array}$ & $\begin{array}{l}\text { - Preferência pelo tratamento não cirúrgico nos casos ortopédicos limítrofes para tratamento cirúrgico e não } \\
\text { - Prúrgico; } \\
\text { - Preferência por imobilização com órteses em vez de talas gessadas ou gesso, visando melhor higienização. } \\
\text { - Orientação por escrito e verbal aos pacientes sobre a evolução da lesão, início de movimento, carga e } \\
\text { reabilitação, visando diminuição do retorno por dúvidas e outros motivos; } \\
\text { - Realização de reduções de fraturas e pequenos procedimentos no próprio PS sob condições controladas de } \\
\text { assepsia, antissepsia e campos estéreis, em detrimento de internação e intervenção no centro cirúrgico; } \\
\text { - Agendamento de cirurgias em regime hospital-dia, quando possível. }\end{array}$ \\
\hline Ambulatório & - As mesmas medidas do Pronto-Socorro. \\
\hline Enfermaria & - Agendamento de cirurgias em regime hospital-dia, quando possível. \\
\hline $\begin{array}{l}\text { ro } \\
\text { gico }\end{array}$ & $\begin{array}{l}\text { - Suspensão das cirurgias eletivas por tempo indeterminado; } \\
\text { - Manutenção das cirurgias de emergência, urgência e neoplasias que necessitam de intervenção precoce; } \\
\text { - Realização das cirurgias o mais rápido possível e com o menor número de profissionais em sala; } \\
\text { - Atenção ao uso de dispositivos como martelo ou fresas que possam espalhar fluidos corporais. Estes } \\
\text { procedimentos devem ser realizados com a técnica apurada, utilizando lâminas e brocas afiadas, além das } \\
\text { - Auperfícies a serem utilizadas mantidas limpas do sangue com auxílio de aspirador. } \\
\text { quaçãa ao uso de bisturi elétrico devido à liberação de aerossóis pela geração de calor. O bisturi elétrico, } \\
\text { quando não puder ser evitado, inclusive para dissecções, precisa estar regulado para sua menor potência, } \\
\text { mantendo a eficiência desejada; } \\
\text { - Não sepultamento de fixações percutâneas, de forma que permita a sua retirada futura em ambulatórios, } \\
\text { evitando mais um procedimento no centro cirúrgico. }\end{array}$ \\
\hline
\end{tabular}

- Número provas didáticas suspensas; e

- Número provas didáticas realizadas.

As intervenções do protocolo 3 foram avaliadas pelo número de procedimentos cirúrgicos realizados, divididos em cirurgias de urgência e eletivas. Para fins de análise dos dados, no nosso serviço, todas as cirurgias que ocorrem após 24 horas de internação são consideradas procedimentos eletivos.

\section{Resultados}

\section{Protocolo 1}

O número de atendimentos no PS e nos ambulatórios caiu $74,1 \%$ e $60,7 \%$, respectivamente, entre os períodos estudados.
A - Tabela 5 e as - Figuras $\mathbf{1}$ e $\mathbf{2}$ resumem os dados nesses dois setores.

Quanto à variação do volume de pacientes internados na disciplina de Ortopedia, no período A, a média diária de leitos ocupados foi de 21,8 (máximo: 30; mínimo: 16) representando $87 \%$ de ocupação. No período $\mathrm{B}$, a média diária de leitos ocupados pela ortopedia foi de 16 (máximo: 23; mínimo: 10), representando $71,4 \%$ de ocupação.

No período estudado foram notificados dois casos suspeitos, que permaneceram em isolamento na enfermaria da Ortopedia; um caso confirmado, que foi transferido para a enfermaria da disciplina de Infectologia; e nenhum óbito. Dos 250 membros do DOT, 6 foram confirmados com COVID-19, sendo 2 destes, residentes. Outros 12 foram afastados por suspeita, sendo 5 destes, residentes. Somente um dos membros do 
Tabela 4 Orientação de equipamento de proteção individual (EPI) para cada setor

\begin{tabular}{|l|l|}
\hline Setor & EPI \\
\hline $\begin{array}{l}\text { Pronto-Socorro } \\
\text { e Ambulatório }\end{array}$ & $\begin{array}{l}\text { - Roupa privativa; } \\
\text { - Avental descartável; } \\
\text { - Óculos ou faceshield; } \\
\text { - Luvas; } \\
\text { - Máscara cirúrgica. }\end{array}$ \\
\hline Enfermaria & $\begin{array}{l}\text { - Roupa privativa; } \\
\text { - Avental descartável; } \\
\text { - Óculos; } \\
\text { - Luvas; } \\
\text { - Touca; } \\
\text { - Máscara cirúrgica ou N95 } \\
\end{array} \quad \begin{array}{l}\text { (N95 obrigatória no atendimento de } \\
\text { pacientes suspeitos ou confirmados). }\end{array}$ \\
\hline $\begin{array}{l}\text { Centro } \\
\text { cirúrgico }\end{array}$ & $\begin{array}{l}\text { - Avental privativa; } \\
\text { - Óculos ou faceshield; } \\
\text { - Luvas; } \\
\text { - Touca; } \\
\text { - Máscara N95. }\end{array}$ \\
\hline
\end{tabular}

DOT confirmados apresentou quadro grave de dispneia, e permaneceu sete dias em UTI antes da alta domiciliar.

No período A, a visita acadêmica na enfermaria contava com nove indivíduos: dois chefes de plantão, cinco residentes do primeiro ano de Ortopedia e Traumatologia (R1), um residente do segundo ano (R2), e um especializando em subárea de Ortopedia e Traumatologia. No período B, a visita diminuiu para seis indivíduos: dois chefes de plantão, dois R1, um R2, e um especializando em subárea de Ortopedia e Traumatologia.

Considerando a adequação completa aos protocolos de EPI no PS, foram necessários dois dias até que a equipe de plantão recebesse de forma integral todos os equipamentos. Nesses dois dias, houve menor quantidade de óculos ou faceshields do que plantonistas, mas os demais EPIs estavam disponíveis. Nesse período, somente os residentes com EPI completo tiveram contato direto com os pacientes.

\section{Protocolo 2}

No total, 18 TAEs do DOT, que realizam atendimento médico ao paciente no hospital referência, foram treinados para o atendimento e manejo de síndromes gripais de baixa complexidade e COVID-19.
Quanto à reorganização da escala de plantão no PS, ela passou de três R1, dois R2, um R3 no período A para dois R1, um R2, e um R3 no período B. Os profissionais dispensados ficaram de sobreaviso, caso fosse necessário comparecimento para auxiliar em procedimentos cirúrgicos ou atendimento médico.

Em relação ao número semanal de aulas para residência e reuniões científicas, houve mudança e adequação do modo presencial para videoconferência. Utilizaram-se os aplicativos Google Hangout Meets (Google, Inc., Mountain View, CA, EUA) ou Zoom (Zoom Video Comunication, Inc., San Jose, CA, EUA). Os membros do DOT receberam orientações sobre o uso dos aplicativos por meio de cartilha produzida pelo CGC/COVID-19 e divulgada pelo aplicativo WhatsApp. Essa adequação demorou uma semana. Após esse período de definição da plataforma, orientação técnica e adesão, as aulas para a residência mantiveram o número de 16 por semana; já as reuniões científicas diminuíram de 10 para 7 por semana. Apenas uma prova didática foi cancelada no período $B$, sendo realizadas duas provas por meio do Socrative (Showbie, Inc., Edmonton, Canadá) associado ao Google Meets ou Zoom.

\section{Protocolo 3}

O número de cirurgias diminuiu $65,8 \%$ entre os períodos estudados. 0 número de cirurgias eletivas e de urgência diminuíram, respectivamente, 71,7\% e 52,2\%. A - Tabela 5 e a - Figura 3 resumem os dados do centro cirúrgico.

\section{Discussão}

O CGC COVID-19 tornou-se um grupo de referência não só para o Departamento de Ortopedia, como também para outros departamentos e para a diretoria do hospital. Assim, centralizou a passagem de informações e auxiliou nas tomadas de decisão referentes à pandemia.

\section{Protocolo 1}

Os atendimentos no PS apresentaram redução importante no período B. Este resultado é um reflexo das medidas tomadas pelo Governo do Estado e pela Prefeitura, que instituíram o isolamento social, ${ }^{6,7}$ e não reflete diretamente as medidas tomadas pela implementação dos protocolos. Supõe-se que os usuários passaram a procurar o PS majoritariamente em casos de urgência. Os membros do departamento também contribuíram para essa redução do fluxo, ao orientar os

Tabela 5 Número de atendimentos e cirurgias antes e depois da implementação dos protocolos

\begin{tabular}{|c|c|c|c|c|c|}
\hline \multirow{2}{*}{\multicolumn{2}{|c|}{\begin{tabular}{|l|} 
\\
Setor
\end{tabular}}} & \multicolumn{2}{|c|}{ Período A } & \multicolumn{2}{|c|}{ Período B } \\
\hline & & Total & Média/dia (mínimo-máximo) & Total & Média/dia (mínimo-máximo) \\
\hline \multicolumn{2}{|c|}{$\begin{array}{l}\text { Atendimentos no Pronto- } \\
\text { Socorro }\end{array}$} & 1.136 & $81,1(38-110)$ & 294 & $21(10-48)$ \\
\hline \multicolumn{2}{|c|}{$\begin{array}{l}\text { Atendimentos } \\
\text { ambulatoriais }\end{array}$} & 1.575 & $157,5(81-209)$ & 619 & $61,9(30-123)$ \\
\hline \multirow[t]{3}{*}{ Cirurgias } & Eletivas & 74 & $5,3(0-8)$ & 21 & $1,5(0-8)$ \\
\hline & Urgências & 32 & $2,3(0-3)$ & 15 & $1,1(0-3)$ \\
\hline & Total & 106 & $7,6(2-13)$ & 36 & $2,6(0-10)$ \\
\hline
\end{tabular}


274 Protocolo de intervenção para enfrentamento da pandemia de COVID-19 Lima et al.

\section{PERIODOA PERIODOB}

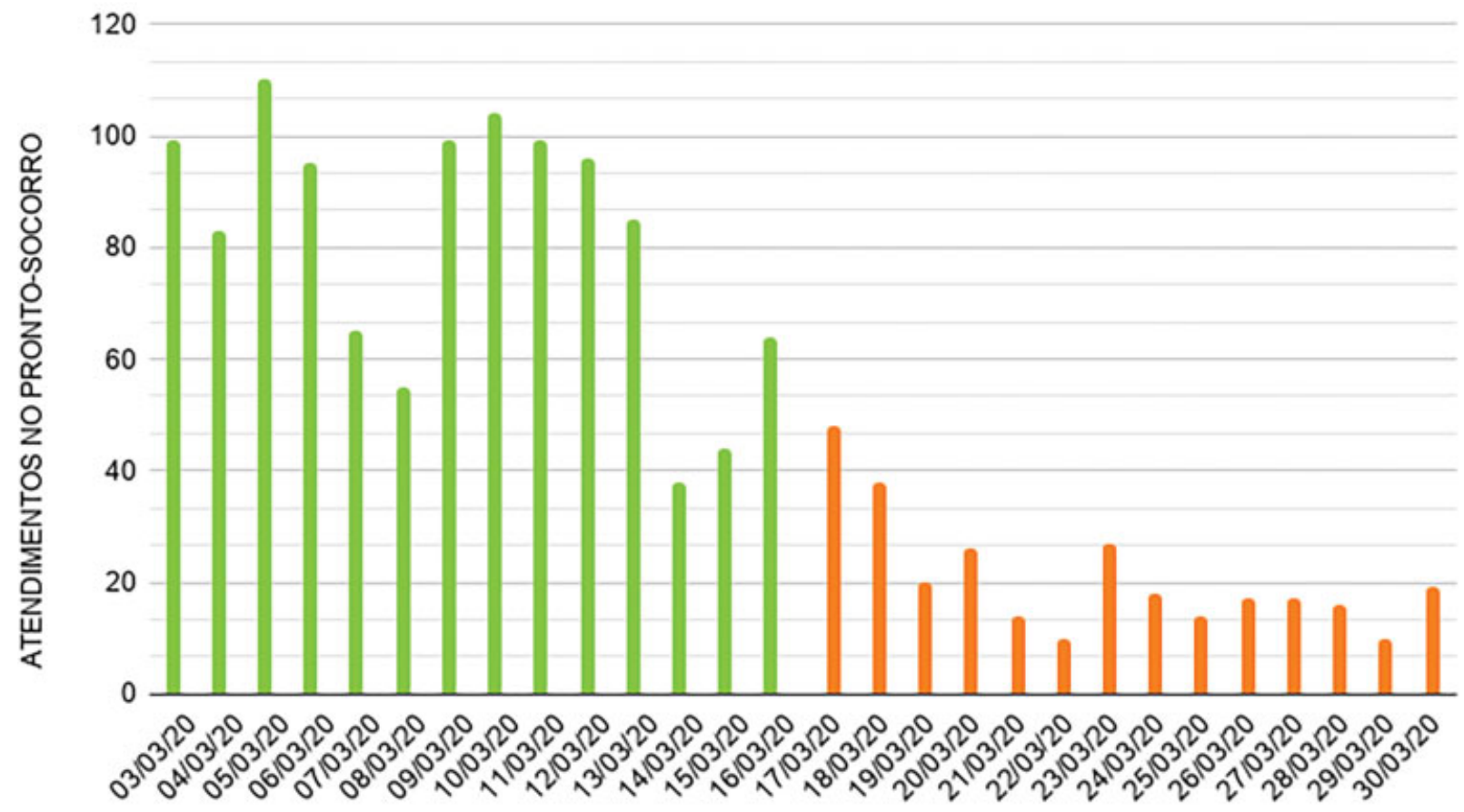

Fig. 1 Gráfico representando número de pacientes atendidos no pronto-socorro de ortopedia do Hospital São Paulo (HSP) ao longo dos dois períodos estudados.

PERIODOA PERIODOB

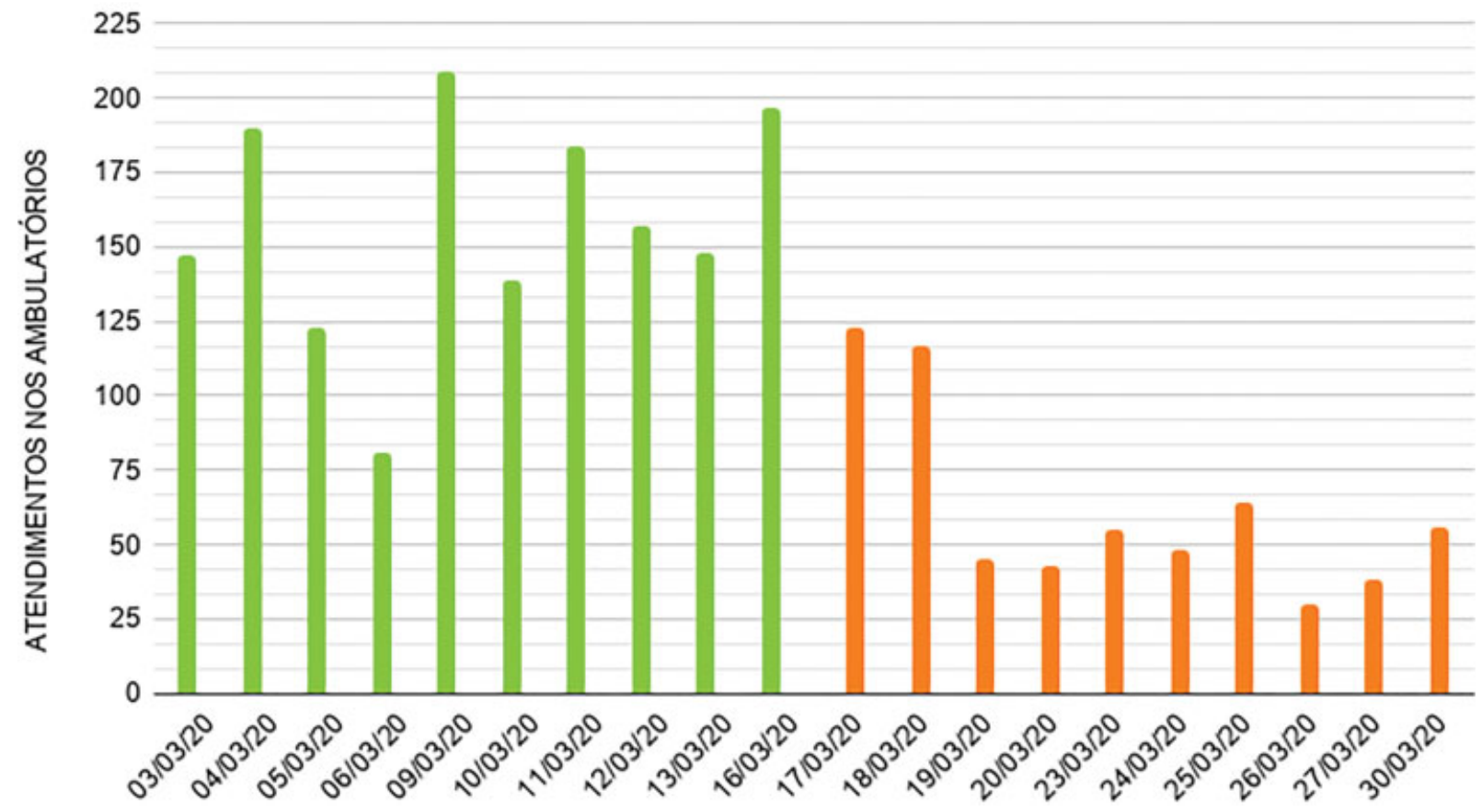

Fig. 2 Gráfico representando número de pacientes atendidos nos ambulatórios de ortopedia do HSP ao longo dos dois períodos estudados.

pacientes e acompanhantes sobre a importância do isolamento social.

Nos ambulatórios, a redução do número de atendimentos teve participação direta da equipe, com a remarcação de consultas não urgentes e priorização das consultas de pós- operatórios recentes. A - Figura 2 mostra essa redução, com os dois primeiros dias do período $\mathrm{B}$ apresentando ainda número elevado de atendimentos. Isso é devido à dificuldade de cancelar e remarcar consultas sem antecedência. Nos dias subsequentes, nota-se importante diminuição no número de atendimentos. 


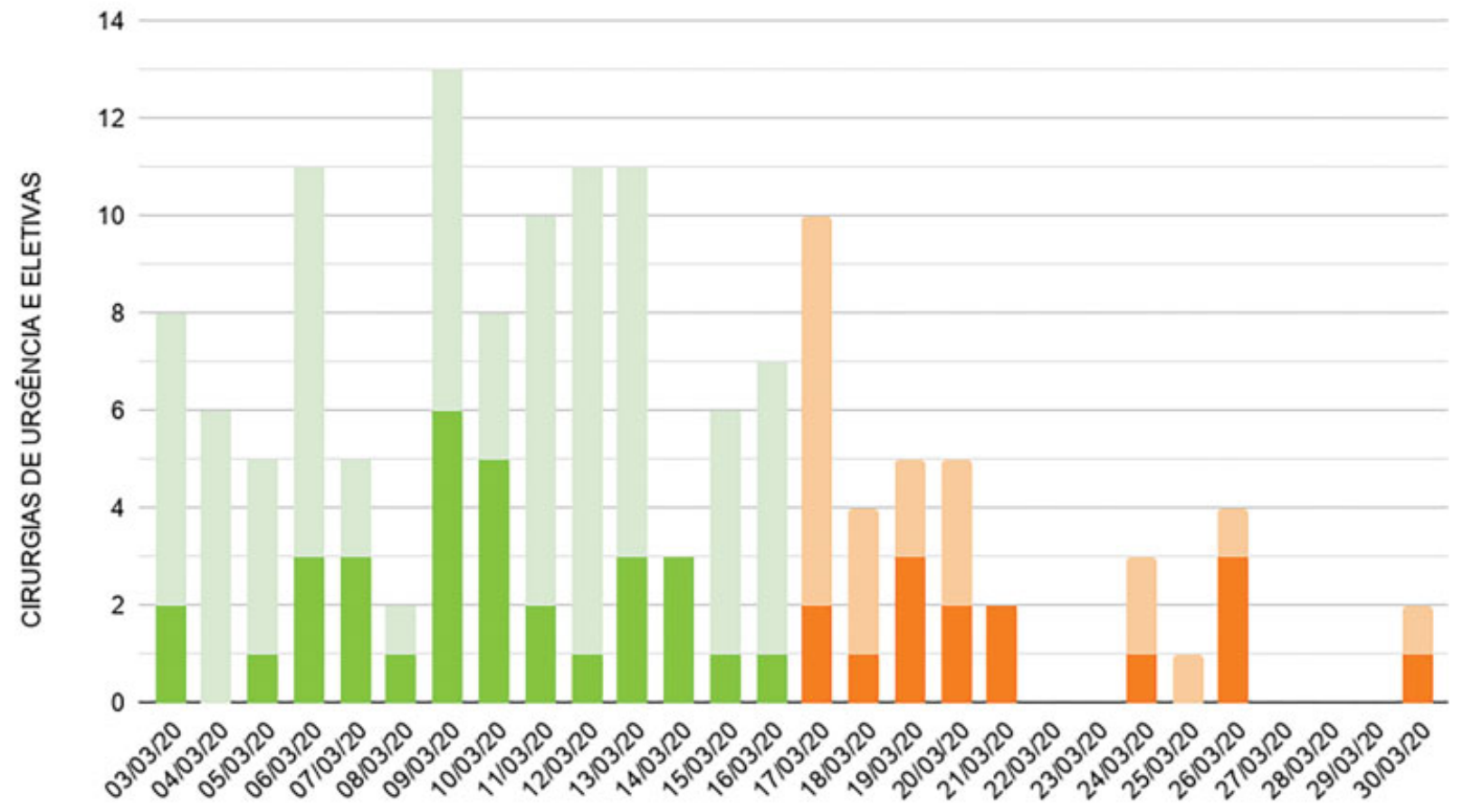

Fig. 3 Gráfico representando número de cirurgias eletivas e de urgência do departamento de ortopedia do HSP ao longo dos dois períodos estudados.

Apesar das medidas do protocolo 1, 6 pessoas da equipe contraíram a doença e outras 12 foram afastadas com suspeita. No entanto, esses dados não indicam uma falha do protocolo, pois não é possível definir se a contaminação desses profissionais foi no hospital estudado, em outro hospital, ou em ambiente extra-hospitalar. Além disso, devido ao período de incubação da doença, existe a possibilidade de a contaminação ter ocorrido antes do início da implementação do protocolo.

Os profissionais de saúde são os protagonistas nessa batalha, e estão nas linhas de frente, sofrendo diretamente as consequências da pandemia. Além do constante risco de contaminação e de transmitir a doença para outras pessoas, os profissionais da saúde estão em um momento de grande estresse mental, e devem ser cuidados nessa questão também. ${ }^{12}$

Um estudo ${ }^{13}$ realizado com ortopedistas infectados com COVID-19 na cidade de Wuhan, na China, mostrou que, dos 24 infectados, 15 precisaram ser hospitalizados. Esse mesmo estudo indicou que o local mais provável de transmissão da doença é a enfermaria, e entre os principais fatores de risco para contaminação foram citados o não uso de máscara N95, falta de EPI adequado, e medidas de higiene inadequadas. A partir disso, o CGC COVID-19 priorizou a distribuição de máscaras N95 e demais EPIs a todos os residentes.

Na Itália, 20\% dos profissionais da saúde foram infectados, e alguns morreram. Relatos médicos descrevem exaustão física e mental, os dilemas de uma triagem difícil, e a dor de perder pacientes e colegas, além do risco de infecção. "Diferentemente de respiradores e leitos de UTI, profissionais da saúde não podem ser construídos na urgência e não podem funcionar $100 \%$ por longos períodos de tempo."17
Acreditamos que a implementação rápida dos protocolos em período anterior ao pico de transmissão e de doentes internados possa ser fator determinante para o preparo de toda a equipe, tanto do ponto de vista técnico quanto também emocional, tendo maior eficiência do que aplicados e construídos na urgência durante os piores períodos da crise.

\section{Protocolo 2}

Apesar de apenas dezoito TAEs terem participado do treinamento ministrado pela disciplina de Infectologia, o CGC/COVID-19 forneceu ao resto da equipe artigos, estudos e cursos online a respeito do COVID-19. Independente da essência cirúrgica da ortopedia, é imprescindível o treinamento dos membros do departamento para enfrentar a pandemia. Outros serviços já estão convocando ortopedistas e outras especialidades para atender na linha de frente contra a pandemia, o que reforça a necessidade de treinamento da equipe.

A reorganização da escala de plantão dos residentes foi uma estratégia para adequar o serviço ao cenário da pandemia sem prejudicar o atendimento, pois a demanda em todos os setores do hospital caiu. Com dois residentes a menos por plantão, mas mantendo pelo menos um residente de cada ano, a estrutura de ensino e troca de experiência é mantida, além de diminuir o risco de contaminação.

Nosso departamento tem um programa extenso de ensino, sendo desafiadora a adequação das atividades acadêmicas. Poucas reuniões foram canceladas e as aulas para residência foram executadas em sua totalidade. As videoconferências das aulas e das reuniões dos diversos grupos do DOT estão inclusive sendo disponibilizadas para todos, o que 
permite maior interação entre os membros da equipe e amplia as oportunidades de aprendizado. Quanto às provas, cada disciplina tem uma forma de avaliar os residentes, e, no período estudado, ocorreriam três provas. Duas foram realizadas por videoconferência, e a outra, de propedêutica geral, foi cancelada enquanto a preceptoria analisa qual é a melhor forma de aplicá-la diante da pandemia.

De forma geral, a carga horária de atividades práticas diminuiu, mas a programação de aulas teóricas foi mantida, com oportunidade de aulas extras. No entanto, ainda é cedo para definir se essas adequações no modo de ensino vão ter impacto no aprendizado dos residentes e especializandos.

\section{Protocolo 3}

Foram instituídas medidas para a redução do número de cirurgias ortopédicas eletivas a fim de minimizar o contato intra-hospitalar de pacientes sem COVID-19 com casos suspeitos ou confirmados. A redução dos procedimentos cirúrgicos possivelmente sofreu influência da diminuição da demanda gerada pelo isolamento social e da opção pelo tratamento não cirúrgico nos casos limítrofes de conduta cirúrgica ou não cirúrgica.

Habitualmente, no nosso serviço, as cirurgias eletivas representam parte considerável do número total de cirurgias. É possível ver na - Tabela 5 e na - Figura 3 que, no período $\mathrm{B}$, o número total de cirurgias diminuiu de forma considerável, principalmente à custa da redução no número de cirurgias eletivas. No dia da implementação dos protocolos, as cirurgias eletivas não foram canceladas; por isso o número elevado de cirurgias em 17 de março. No entanto, nos dias seguintes, notam-se os resultados dos protocolos, com a diminuição expressiva do número de cirurgias.

Essas ações permitiram o redirecionamento dos recursos do hospital para casos mais graves, além de liberar os leitos mais rapidamente, diminuindo o risco de propagação do vírus na enfermaria, e permitindo a realocação de pacientes suspeitos para COVID-19 em leitos isolados.

\section{Adaptação de Outras Especialidades à Pandemia}

Já é possível ver na literatura alguns artigos ${ }^{18-20}$ que relatam a experiência de outras especialidades frente à pandemia, sugerindo medidas para adequação dos serviços médicos neste cenário. Urologistas e dermatologistas estão tomando medidas semelhantes às nossas, com suspensão das cirurgias eletivas, diminuição do fluxo de pacientes nos ambulatórios, uso adequado de EPIs, entre outras. ${ }^{18-20}$ Anestesiologistas já estão adaptando seus procedimentos e equipamentos para atender pacientes com COVID-19, além de sugerir novas rotinas para os centros cirúrgicos. ${ }^{14-16}$ Esperamos que nosso relato e nossas sugestões de medidas de intervenção auxiliem outros serviços de ortopedia e estimulem outras especialidades a compartilhar suas experiências e desenvolver novos protocolos para combater essa pandemia.

\section{Limitações do Estudo}

Este estudo analisou comparativamente um curto período de tempo entre a implementação do protocolo e o período prépandemia. Dessa forma, ainda não é possível saber os impac- tos em longo prazo de tais medidas, sendo necessária a realização de pesquisas futuras.

As atividades do departamento não ocorrem somente no hospital apresentado no estudo. 0 departamento tem uma atuação multicêntrica em diversos hospitais mantendo sua coordenação central no hospital estudado. A experiência e os conhecimentos gerados no hospital referência foram, em parte, expandidos para os hospitais afiliados, mas o impacto dessas mudanças nesses locais não foi avaliado neste estudo.

\section{Conclusões}

Este estudo demonstrou a viabilidade e eficiência da rápida implementação de protocolos de intervenção em meio ao cenário de pandemia por COVID-19. Com a adaptação das atividades do departamento, os protocolos reduziram o risco de contágio dos pacientes e profissionais de saúde. Sua aplicação manteve as atividades assistenciais, acadêmicas e científicas funcionantes, apesar da priorização das medidas de enfrentamento à COVID-19.

\section{Conflito de Interesses}

Os autores declaram não haver conflito de interesses.

\section{Agradecimentos}

Os autores agradecem ao Dr. Flavio Faloppa e aos integrantes do Comitê Gestor de Crise COVID-19: Carlos Eduardo Franciozi, Fabio Teruo Matsunaga, Gustavo Tadeu Sanchez, Marcel Jun Sugawara Tamaoki e Renato Hiroshi Salvioni Ueta. Todos, além de contribuir para a atuação do comitê, foram indispensáveis na elaboração deste trabalho.

\section{Referências}

1 World Health Organization. Pneumonia of unknown cause China [acesso em: 05 de janeiro, 2020]. Disponível em: https:// www.who.int/csr/don/05-january-2020-pneumonia-of-unkown -cause-china/en/

2 World Health Organization. Novel Coronavirus - China [acesso em: 12 de janeiro, 2020]. Disponível em: https://www.who.int/csr/don/12-january-2020-novel-coronavirus-china/en/

3 World Health Organization. Rolling updates on coronavirus disease (COVID-19). 2020. Disponível em: https://www.who.int/emergencies/diseases/novel-coronavirus-2019/events-as-they-happen

4 Worldometer. COVID-19 coronavirus pandemic [acesso em: 30 de março, 2020]. Disponível em: https://www.worldometers.info/ coronavirus/

5 Ministério da Saúde (Brasil). Brasil confirma primeiro caso da doença [acesso em: 26 de fevereiro, 2020]. Disponível em: https://www.saude.gov.br/noticias/agencia-saude/46435-brasilconfirma-primeiro-caso-de-novo-coronavirus

6 Prefeitura de São Paulo. Prefeitura de São Paulo declara situação de emergência com medidas para evitar disseminação do coronavírus [acesso em: 17 de março, 2020]. Disponível em: http:// www.capital.sp.gov.br/noticia/prefeitura-de-sao-paulo-declarasituacao-de-emergencia-com-medidas-para-evitar-disseminacao-do-coronavirus

7 Governo do Estado de São Paulo. Governo de São Paulo anuncia estado de calamidade pública em território paulista [acesso em: 20 de março, 2020]. Disponível em: https://www.saopaulo.sp. gov.br/spnoticias/governo-de-sp-anuncia-novas-medidas-paraintensificar-o-combate-ao-coronavirus/ 
8 World Health Organization. Infection prevention and control during health care when novel coronavirus (ncov) infection is suspected. 2020. Disponível em: https://www.who.int/publications-detail/ infection-prevention-and-control-during-health-care-when-novel -coronavirus-(ncov)-infection-is-suspected-20200125

9 Conselho Federal de Medicina. Posição do Conselho Federal de Medicina sobre a pandemia de COVID-19: contexto, análise de medidas e recomendações [acesso em: 17 de janeiro, 2020]. Disponível em: http://portal.cfm.org.br/index.php?option=com_content \&view=article\&id=28634

10 British Orthopaedic Association. Information for BOA members on trauma and othopaedic care in the UK during coronavirus pandemic [acesso em: 24 de março, 2020]. Disponível em: https://www.boa.ac.uk/resources/statement-for-boa-memberson-trauma-and-orthopaedic-care-in-the-uk-during-coronavirus -pandemic.html

11 Chang Liang Z, Wang W, Murphy D, Po Hui JH. Novel Coronavirus and Orthopaedic Surgery: Early Experiences from Singapore. [publicado online antes da impressão, 2020 Mar 20]J Bone Joint Surg Am 2020; 10: [Epub ahead of print] . Doi: 10.2106/JBJS.20.00236

12 Wang H, Wang S, Yu K. COVID-19 infection epidemic: the medical management strategies in Heilongjiang Province, China. Crit Care 2020;24(01):107

13 Guo X, Wang J, Hu D, et al. The orthopaedic forum survey of COVID19 disease among orthopaedic surgeons in Wuhan, Peoples's Republic of China. J Bone Joint Surg 2020. Disponível em:https:// journals.lww.com/jbjsjournal/Documents/Guo_20.00417.pdf
14 Wong J, Goh QY, Tan Z, et al. Preparing for a COVID-19 pandemic: a review of operating room outbreak response measures in a large tertiary hospital in Singapore. [publicado online antes da impressão, 2020 Mar 11]Can J Anaesth 2020; [Epub ahead of print] . Doi: 10.1007/s12630-020-01620-9

15 Ti LK, Ang LS, Foong TW, Ng BSW. What we do when a COVID-19 patient needs an operation: operating room preparation and guidance. [publicado online antes da impressão, 2020 Mar 6]Can J Anaesth 2020; [Epub ahead of print] . Doi: 10.1007/s12630-020-01617-4

16 Wax RS, Christian MD. Practical recommendations for critical care and anesthesiology teams caring for novel coronavirus (2019-nCoV) patients. Can J Anaesth 2020;67(05):568-576; [Epub ahead of print]. Doi: 10.1007/s12630-020-01591-x

17 The Lancet. COVID-19: protecting health-care workers. Lancet 2020;395(10228):922

18 Nowroozi A, Amini E. Urology practice in the time of COVID-19. [publicado online antes da impressão, 2020 Mar 24]Urol J 2020. Doi: 10.22037/uj.v0i0.6065

19 Chan MC, Yeo SEK, Chong YL, Lee YM. Stepping Forward: Urologists' Efforts During the COVID-19 Outbreak in Singapore. [publicado online antes da impressão, 2020 Mar 17]Eur Urol 2020;S0302-2838 (20)30145-7 S0302-2838(20)30145-30147; [Epub ahead of print]

20 Radi G, Diotallevi F, Campanati A, Offidani A. Global coronavirus pandemic (2019-nCOV): Implication for an Italian medium size dermatological clinic of a ii level hospital. [publicado online antes da impressão, 2020 Mar 22]J Eur Acad Dermatol Venereol 2020; [Epub ahead of print] . Doi: $10.1111 / j d v .16386$ 